\title{
Parietal Stimulation Decouples Spatial and Feature-Based Attention
}

\author{
Bertram Schenkluhn, ${ }^{1,2}$ Christian C. Ruff,, ${ }^{1,2}$ Klaartje Heinen, ${ }^{1,2,3}$ and Christopher D. Chambers ${ }^{1,2,3}$ \\ ${ }^{1}$ Institute of Cognitive Neuroscience, University College London, London WC1N 3AR, United Kingdom, ${ }^{2}$ Wellcome Department of Imaging Neuroscience, \\ University College London, London WC1N 3BG, United Kingdom, and ${ }^{3}$ School of Psychology, Cardiff University, Cardiff CF10 3AT, United Kingdom
}

Everyday visual scenes contain a vast quantity of information, only a fraction of which can guide our behavior. Properties such as the location, color and orientation of stimuli help us extract relevant information from complex scenes (Treisman and Gelade, 1980; Livingstone and Hubel, 1987). But how does the brain coordinate the selection of such different stimulus characteristics? Neuroimaging studies have revealed significant regions of overlapping activity in frontoparietal cortex during attention to locations and features, suggesting a global component to visual selection (Vandenberghe et al., 2001; Corbetta and Shulman, 2002; Giesbrecht et al., 2003; Slagter et al., 2007). At the same time, the neural consequences of spatial and feature-based attention differ markedly in early visual areas (Treue and Martinez-Trujillo, 2007), implying that selection may rely on more specific top-down processes. Here we probed the balance between specialized and generalized control by interrupting preparatory attention in the human parietal cortex with transcranial magnetic stimulation (TMS). We found that stimulation of the supramarginal gyrus (SMG) impaired spatial attention only, whereas TMS of the anterior intraparietal sulcus (aIPS) disrupted spatial and feature-based attention. The selection of different stimulus characteristics is thus mediated by distinct top-down mechanisms, which can be decoupled by cortical interference.

Key words: attention; transcranial magnetic stimulation; parietal cortex; spatial selection; feature-based selection; top-down control

\section{Introduction}

Selective attention is crucial for optimizing the limited capacity of the human visual system (Broadbent, 1958; Kastner and Ungerleider, 2000). Contemporary theories suggest that selection influences sensory competition by biasing neural processing in favor of behaviorally relevant stimuli (Desimone and Duncan, 1995; Duncan et al., 1997). Hence, when we attend to a particular location or feature (e.g., color), behavioral and neuronal responses to stimuli that share the selected property are enhanced (Corbetta et al., 1990; Heinze et al., 1994; Chawla et al., 1999; Giesbrecht et al., 2006; Schoenfeld et al., 2007). These amplified neural representations are believed to result from top-down control signals biasing "bottom-up" sensory processing (Desimone and Duncan, 1995; Kastner and Ungerleider, 2000; Corbetta and Shulman, 2002; Yantis and Serences, 2003; Maunsell and Treue, 2006).

Several studies have used cued attention paradigms (Posner et al., 1980) in combination with functional magnetic resonance imaging (fMRI) to identify the neural sources of attentional control. Such experiments typically report the engagement of a frontoparietal network during visual attention to locations (Corbetta et al., 2000, 2005; Hopfinger et al., 2000; Woldorff et al., 2004; Slagter et al., 2007) or features (Shulman et al., 1999; Vanden-

\footnotetext{
Received July 30, 2008; accepted Sept. 6, 2008.

K.H. and C.D.C. are supported by a David Phillips Felowship from the Biotechnology and Biological Sciences Research Council (C.D.C.). C.C.R. is supported by the Medical Research Council (UK). We thank Jon Driver for helpful discussions.

Correspondence should be addressed to Christopher D. Chambers, School of Psychology, Cardiff University, Cardiff CF10 3AT, UK. E-mail: chambersc1@cardiff.ac.uk.

D0I:10.1523/JNEUROSCI.3591-08.2008

Copyright $\odot 2008$ Society for Neuroscience $\quad$ 0270-6474/08/2811106-05\$15.00/0
}

berghe et al., 2001; Giesbrecht et al., 2003; Luks and Simpson, 2004; Slagter et al., 2007). Importantly, the majority of such activations appear common to spatial and feature-based conditions, suggesting that selection may be overseen by a generalized topdown mechanism (see also Slagter et al., 2005a).

In contrast to evidence for global attentional control processes in frontoparietal cortex, human fMRI and primate neurophysiological studies have shown that the effects of spatial and featurebased attention differ substantially in the visual cortex (Saenz et al., 2002; Serences and Boynton, 2007; Treue and MartinezTrujillo, 2007). During spatial selection, for instance, only neurons whose spatial receptive fields correspond with the current focus of attention show enhanced activity (Womelsdorf et al., 2006). For feature-based attention, however, the responses of neurons tuned to the attended feature are enhanced across the entire visual field (Saenz et al., 2002; Serences and Boynton, 2007). These distinct outcomes imply that the selection of spatial and feature-based information may depend on more specialized, rather than generalized, top-down processes.

In the present study, we used the convergent technique of transcranial magnetic stimulation (TMS) to test whether common cortical regions are necessary for spatial and feature-based selection. Participants undertook a visual search task in which a precue indicated either the location or color (feature) of an upcoming target stimulus. To interrupt preparatory attention, we applied TMS during the cue presentation to one of three parietal areas previously implicated in the strategic control of visual selection, including the supramarginal gyrus (SMG), the anterior IPS, and the posterior IPS of the right hemisphere (Vallar and Perani, 1986; Corbetta et al., 2000; Perry and Zeki, 2000; Vanden- 


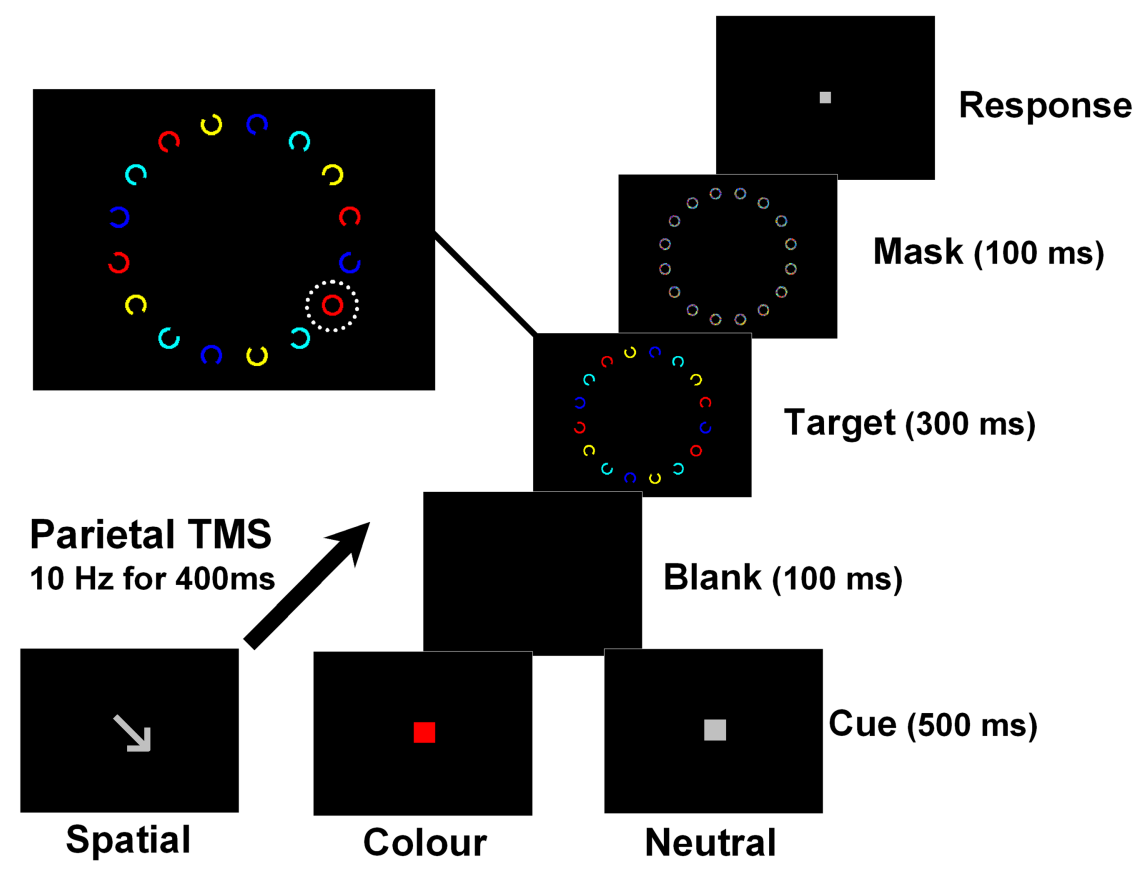

Figure 1. Display sequence in the cued attention task. Each trial commenced with a fixation stimulus, followed by a cue: Spatial (arrow), Feature (colored square), or Neutral (gray square). The neutral cue was noninformative, whereas the spatial and the feature cues were fully informative. After a short blank ( $100 \mathrm{~ms}$ ), a Landolt array appeared in which the target stimulus (full circle) was either present (50\%) or absent (50\%). In the example shown above, a red target occurs in the lower right quadrant (outlined in inset). On each trial, a $400 \mathrm{~ms}$ train of TMS was delivered randomly at either a low or high intensity, beginning $100 \mathrm{~ms}$ after cue onset. Online monitoring of gaze ensured that participants maintained fixation throughout the entire trial.

berghe et al., 2001; Giesbrecht et al., 2003; Macaluso et al., 2003; Chambers et al., 2004b; Slagter et al., 2007). If these regions host an essential global mechanism, then we expected TMS to reduce the perceptual advantage conferred by both spatial and color cues. Alternatively, if spatial and feature-based attention are controlled by separate systems, then parietal TMS was expected to yield dissociable effects within each mode of selection.

\section{Materials and Methods}

Participants. Eleven neurologically healthy right-handed volunteers (aged 20 -30 years, $\mathrm{M}=25, \mathrm{SD}=3.7,7$ females) with normal vision were paid for their participation. Nine participants undertook the behavioral experiment. Eleven participants took part in the subsequent TMS experiment, of which 10 participated in the SMG condition.

Apparatus. All experimental sessions were conducted in a darkened soundproof testing chamber. Visual stimuli were presented at a midsagittal viewing distance of $65 \mathrm{~cm}$, on a 17 inch CRT monitor $(60 \mathrm{~Hz}$ vertical refresh rate; $1024 \times 768$ resolution; black background). Throughout the experiment, the participant's head was fixed with a chin rest, including forehead and temple stabilizers. Gaze was monitored on all trials with an ASL504 High Speed remote infrared eye-tracking system. Trials in which gaze deviated $>2^{\circ}$ from fixation or in which eyeblinks occurred were discarded.

Stimuli and task. In the visual search task (Fig. 1), participants decided whether a target stimulus (closed circle) was present or absent from an array of Landolt stimuli. On each trial, participants were cued to the target location (one of four quadrants) or target color (one of four colors), thus eliminating $75 \%$ of the distractors as potential targets. These cueing conditions were contrasted with a neutral baseline, in which the cue contained no information about the target. Before the TMS study, the effectiveness of this cueing paradigm was confirmed in a separate behavioral experiment (see supplemental material, available at www. jneurosci.org).

Participants responded with the index or middle finger of their righthand to indicate the presence or absence of the target, respectively. Par- ticipants were instructed to respond as accurately as possible, with no emphasis on speed. The distractor array consisted of 16 Landolt stimuli $\left(0.92^{\circ}\right.$ diameter $)$ distributed radially around the center of the screen $\left(5.1^{\circ}\right.$ radius). Four equidistant Landolts were located in each quadrant of the visual field, in one of four different colors (blue, yellow, red, and turquoise). On $50 \%$ of trials, one Landolt stimulus was replaced by a full circle (target stimulus). Target location and color were pseudorandomized and balanced for each quadrant of the display.

Trials were self-initiated and commenced with the onset of the central fixation stimulus (gray square, $0.15^{\circ} \times 0.15^{\circ}$ visual angle, $600-$ $1300 \mathrm{~ms}$ ). Thereafter, a cue appeared for $500 \mathrm{~ms}$, which was either an arrow (spatial cue, $0.5^{\circ} \times$ $0.4^{\circ}$ ) pointing toward one of the four quadrants, a colored square (feature cue, $0.28^{\circ} \times 0.28^{\circ}$ ) indicating one of the four colors, or a gray square (neutral cue, $0.28^{\circ} \times 0.28^{\circ}$ ). Both the spatial and the color cue eliminated $75 \%$ of the distractors as potential targets (either three quadrants or three colors), whereas the neutral cue indicated only that the array was about to be presented. After a short blank, the distractor array was presented for $300 \mathrm{~ms}$, followed by a backward mask for $100 \mathrm{~ms}$. The mask consisted of 16 full circles, each including a random mix of colored pixels that appearing at the same size and location as the preceding distractor array.

Cueing conditions were presented in blocks of 20 trials, with each block containing trials of one attention condition only. The first four trials of each block were discarded to eliminate psychophysical variance associated with task switching. Each experimental run contained 12 such blocks (four per attention condition) presented in a counterbalanced order.

In the initial behavioral experiment, participants undertook 2-3 training runs, followed by one experimental run. Task difficulty was calibrated between runs by adjusting the gap size of the Landolt stimuli to ensure stable individual performance in an appropriate psychophysical range. To guarantee an identical range of target displays across conditions, the gap size was held constant for each cue type.

The subsequent TMS experiment consisted of three separate experimental sessions, each commencing with one run of "Sham TMS" (in which the coil was oriented perpendicular to the scalp, rather than tangential) plus two runs with active TMS. The accumulated results of the initial Sham runs were later analyzed as an additional control experiment (see supplemental material, available at www.jneurosci.org). Within each session, active stimulation was applied to one region only. The stimulated site (SMG, aIPS, pIPS) was blocked between testing sessions and counterbalanced across participants using a balanced Latin square. Consecutive TMS sessions were separated by at least $24 \mathrm{~h}$.

TMS and MRI parameters. A repetitive pulse-train of TMS was delivered on each trial, from 100 to $500 \mathrm{~ms}$ after cue onset (five pulses at 10 $\mathrm{Hz}$ ). The timing of this pulse train was selected to encompass the most likely period during which the parietal cortex would be necessary for controlling spatial and feature-based attention (Anllo-Vento et al., 1998; Slagter et al., 2005a,b). The intensity of stimulation was delivered randomly on a trial-by-trial basis at $40 \%$ (low) or $120 \%$ (high) of individual resting motor threshold.

Target sites for TMS were determined individually for each participant through MR coregistration (Fig. 2). Neuroanatomical definitions for each site were based on previous TMS studies; SMG was defined as in Chambers et al. (2007), whereas aIPS and pIPS were defined as in Morris et al. (2007). Before testing, structural MRI scans of each participant were acquired in a 1.5 Tesla whole-body scanner (SONATA, Siemens) using an 8-channel head-array coil. The anatomical regions of interest were 


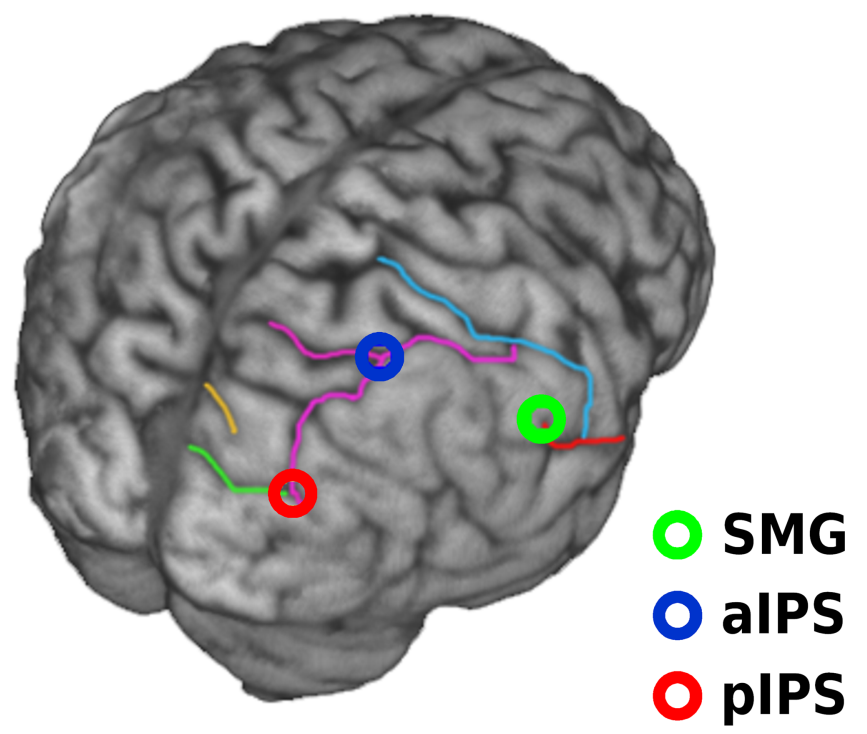

Figure 2. Sites of cortical stimulation. In each session, TMS was applied to one of three cortical sites in the right hemisphere, shown here on the rendered cortical surface of one participant. The highlighted sulci were used to identify the sites of stimulation, including SMG (green circle), alPS (blue circle), and pIPS (red circle). Mean MNI coordinates ( $x, y, z \pm 1$ SD) were $63 \pm 2.5,-40 \pm 5.1,43 \pm 5.4$ for SMG, $33 \pm 6.9,-61 \pm 5.8,64 \pm 4.9$ for alPS, and $25 \pm 5.2,-86 \pm 4.1,42 \pm 8.4$ for pIPS.

then localized individually in 3D-rendered brain images using MRICro imaging software (Rorden and Brett, 2000). At the beginning of each testing session, one region of interest was coregistered to the scalp surface using MR coregistration software (MRIReg) and a magnetic tracking device (miniBIRD 500, Ascension Tech). Stimulation was then applied via a Magstim Super Rapid ${ }^{2}$ system, with the virtual cathode of a figureof-eight coil $(70 \mathrm{~mm})$ positioned over the marked location and the handle oriented toward the vertex. The TMS coil was fixed in position using a clamp and tripod. To minimize distraction caused by coil discharge artifacts, participants wore foam earplugs throughout the experiment.

\section{Results}

The dependent variable in all analyses was the perceptual sensitivity of target detection $\left(d^{\prime}\right)$. We used the neutral condition as a within-subjects and within-session baseline for determining the perceptual advantage conferred by spatial and color cues. To calculate this index, $d^{\prime}$ in the neutral condition was subtracted from $d^{\prime}$ in the spatial and the color condition $\left(\Delta d^{\prime}\right)$.

Separate two-way repeated-measures ANOVAs of $\Delta d^{\prime}$ were undertaken for each site, including the factors of Cue Condition (spatial, color) and TMS Intensity (low, high) (Fig. 3). In each case (SMG, aIPS, pIPS), a significant main effect of Cue Condition was observed (all $p<0.01$ ), reflecting the attentional advantage conferred by color cues relative to spatial cues. This observation was consistent with our initial behavioral experiment (see supplemental material, available at www.jneurosci.org).

For SMG (Fig. 3A), the main effect of TMS Intensity did not reach significance $\left(F_{(1,9)}=2.5, p=0.11\right)$; however, a significant TMS Intensity $\times$ Cue Condition interaction was observed $\left(F_{(1,9)}\right.$ $=6.3, p<.05)$. Analysis of simple main effects revealed that $\mathrm{SMG}$ stimulation significantly reduced the attentional advantage yielded by spatial cues (high-intensity TMS, $\Delta d^{\prime}=0.29$, SE $=$ 0.14 ; low-intensity TMS, $\Delta d^{\prime}=0.65, \mathrm{SE}=0.08 ; p=0.02$ ) but not by color cues $(p=0.84)$. Follow-up analyses and control experiments confirmed that the selective impairment of spatial selection was not attributable to differences in task difficulty or to different degrees of reflexive priming that may have been induced

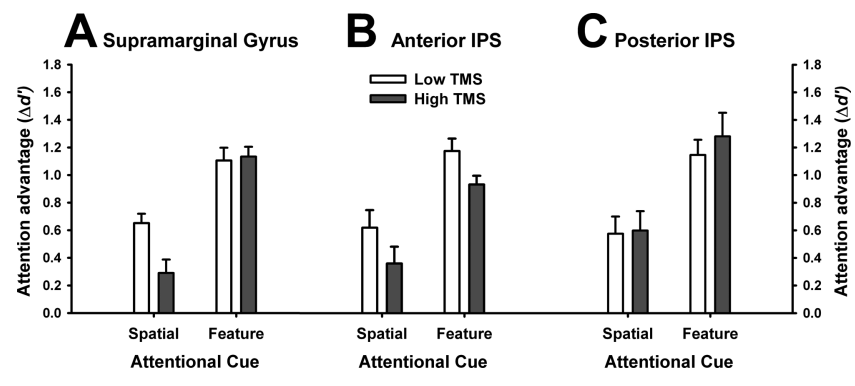

Figure 3. The effect of parietal stimulation on strategic control of spatial and feature-based selection. Each panel indicates the performance advantage conferred by the spatial and feature cues relative to the neutral condition $\left(\Delta d^{\prime}\right)$. Results are plotted according to the cue condition (spatial, feature) and TMS intensity (low, high). $\boldsymbol{A}$, Stimulation of the supramarginal gyrus (SMG) during the cue period impaired goal-directed allocation of spatial attention, but not feature-based attention. $\boldsymbol{B}$, Stimulation of the anterior IPS caused a uniform deficit of both spatial and feature-based attention. C, Stimulation of the posterior IPS did not affect selection of either stimulus dimension. Error bars are $+1 \mathrm{SE}$.

by the cues (see supplemental material, available at www.jneurosci.org).

For aIPS (Fig. $3 B$ ), no significant interaction was observed between TMS Intensity and Cue Condition $\left(F_{(1,10)}=0.005, p=\right.$ 0.94). Instead, a significant main effect of TMS Intensity was detected $\left(F_{(1,10)}=5.5, p=0.04\right)$, reflecting overall reduced performance during high-intensity TMS $\left(\Delta d^{\prime}=0.65, \mathrm{SE}=0.10\right)$ compared with low-intensity TMS $\left(\Delta d^{\prime}=0.90, \mathrm{SE}=0.14\right)$. Thus, stimulation of the aIPS impaired detection performance uniformly in both the spatial and feature-based conditions. For pIPS (Fig. 3C), no significant effects of TMS intensity were found (both $p>0.6$ ).

\section{Discussion}

Our results highlight a functional specialization within the parietal cortex for attention to different stimulus characteristics. Stimulation of the aIPS impaired preparatory spatial- and feature-based selection, whereas TMS of the SMG impaired spatial selection only. Interestingly, stimulation of the pIPS did not disrupt either process.

The role of the SMG observed here is consistent with previous studies demonstrating the involvement of this area in spatial selection (Vallar and Perani, 1986; Perry and Zeki, 2000), especially in vision (Chambers et al., 2004b). The present results further suggest that the attentional role of the SMG may be highly specialized for visuospatial control, without being singularly critical for top-down selection of color information. Our findings thus disconfirm the hypothesis that attention is principally controlled by a global top-down system, and instead substantiate the existence of specialized cortical modules that are anatomically separate yet functionally linked.

The observed disruption of spatial selection during aIPS stimulation accords with the results of several fMRI studies reporting activations in anterior IPS regions during visuospatial cueing (Corbetta et al., 2000; Hopfinger et al., 2000; Giesbrecht et al., 2003). The results further illustrate a critical role of the aIPS in feature-based attention, consistent with fMRI evidence of aIPS activation during attention to motion (Shulman et al., 1999) or color (Vandenberghe et al., 2001; Giesbrecht et al., 2003; Slagter et al., 2007). Considered alongside the specific role of the right SMG, these results imply varying degrees of functional specialization within the parietal lobe for different aspects of visual attention (Giesbrecht et al., 2003; Slagter et al., 2007).

How might the aIPS and SMG coordinate attentional selec- 
tion within the frontoparietal network? One possibility is that the aIPS integrates spatial and feature-based information by coding an abstract salience representation, similar to the lateral intraparietal area in nonhuman primates (Bisley and Goldberg, 2003; Balan and Gottlieb, 2006). To produce this map, aIPS would require convergent inputs from multiple levels of the cortical hierarchy, including regions specialized for processing spatial information. Our results suggest that the SMG may be an important input region to aIPS. If so, then the critical epoch of spatial selection in the SMG should precede that of the aIPS, which could be tested in future TMS studies using single-pulse (Chambers et al., 2004a) or twin-coil methods (Pascual-Leone and Walsh, 2001; Ellison et al., 2007).

Interestingly, stimulation of the pIPS yielded no significant effects on spatial or feature-based selection, suggesting that pIPS is not singularly critical for either process. This functional dissociation between anterior and posterior branches of the IPS implies the existence of functionally distinct subdivisions within this area, as can be assumed from neurophysiological evidence in primates (Grefkes and Fink, 2005; Orban et al., 2006). Stimulation of posterior parietal regions such as the pIPS and angular gyrus has previously been shown to disrupt reflexive shifts of attention (Chambers et al., 2007) and impair performance in visual search paradigms (Rushworth and Taylor, 2006). Because the preparatory attention shifts in the present paradigm are assumed to occur before target onset, one might speculate that the pIPS is more crucial for target-related processing, perhaps maintaining and/or updating spatial representations during attention shifts or saccades. Such a functional dissociation would be consistent with the results of Morris et al. (2007), who found that TMS of pIPS but not aIPS disrupts spatial remapping across eye movements.

Overall, our results indicate that spatial and feature-based selection are controlled by anatomically distinct systems in the parietal cortex, which is arguably inconsistent with the predominance of common activity reported in previous neuroimaging studies (Vandenberghe et al., 2001; Giesbrecht et al., 2003; Slagter et al., 2007). This discrepancy between TMS and fMRI data is predictable given the underlying logic of each method (Chambers and Mattingley, 2005). As noted previously (Chambers et al., 2004b), common activations in fMRI studies cannot be securely attributed to common cognitive processes because it is unclear what proportion of the observed activity is necessary for task performance. For instance, during spatial and feature-based attention, it is conceivable that much overlapping cortical activity reflects the redundant (and ecologically sensible) engagement of distinct spatial and feature-based systems, even if only one such system is required under specific laboratory conditions (Giesbrecht et al., 2003; Slagter et al., 2007). Moreover, because of the limited temporal resolution of BOLD, it can be difficult to distinguish neural responses associated with cues and targets, which could underestimate any differentiation in the anatomical distribution of preparatory activity (Vandenberghe et al., 2001). These caveats highlight the complimentary value of TMS in revealing the functional and temporal specificity of such activations.

A promising avenue for future studies will be to combine TMS with fMRI (Ruff et al., 2006, 2008; Sack et al., 2007), revealing the consequences of SMG/IPS stimulation on target-related activity in retinotopic visual cortex (V1-V5). In particular, the present results raise the question of whether stimulating the aIPS would yield qualitatively different effects in the visual cortex during spatial and color-based selection, consistent with recent evidence that attention depends on top-down connections between parietal and occipital areas (Saalmann et al., 2007).

To conclude, we have shown that the strategic control of spatial and feature-based attention is functionally dissociable between the SMG and anterior IPS of the right parietal cortex. Furthermore, our findings indicate that cortical populations in the dorsal visual stream can be crucial for nonspatial selection. Overall, these conclusions accord with existing evidence for distinct consequences of spatial and feature-based attention in the visual cortex (Saenz et al., 2002; Serences and Yantis, 2007; Treue and Martinez-Trujillo, 2007), and suggest that such effects are likely to stem from distinct mechanisms of top-down control.

\section{References}

Anllo-Vento L, Luck SJ, Hillyard SA (1998) Spatio-temporal dynamics of attention to color: evidence from human electrophysiology. Hum Brain Mapp 6:216-238.

Balan PF, Gottlieb J (2006) Integration of exogenous input into a dynamic salience map revealed by perturbing attention. J Neurosci 26:9239-9249.

Bisley JW, Goldberg ME (2003) Neuronal activity in the lateral intraparietal area and spatial attention. Science 299:81-86.

Broadbent D (1958) Perception and communication. London: Pergamon.

Chambers CD, Mattingley JB (2005) Neurodisruption of selective attention: insights and implications. Trends Cogn Sci 9:542-550.

Chambers CD, Payne JM, Stokes MG, Mattingley JB (2004a) Fast and slow parietal pathways mediate spatial attention. Nat Neurosci 7:217-218.

Chambers CD, Stokes MG, Mattingley JB (2004b) Modality-specific control of strategic spatial attention in parietal cortex. Neuron 44:925-930.

Chambers CD, Payne JM, Mattingley JB (2007) Parietal disruption impairs reflexive spatial attention within and between sensory modalities. Neuropsychologia 45:1715-1724.

Chawla D, Rees G, Friston KJ (1999) The physiological basis of attentional modulation in extrastriate visual areas. Nat Neurosci 2:671-676.

Corbetta M, Shulman GL (2002) Control of goal-directed and stimulusdriven attention in the brain. Nat Rev Neurosci 3:201-215.

Corbetta M, Miezin FM, Dobmeyer S, Shulman GL, Petersen SE (1990) Attentional modulation of neural processing of shape, color, and velocity in humans. Science 248:1556-1559.

Corbetta M, Kincade JM, Ollinger JM, McAvoy MP, Shulman GL (2000) Voluntary orienting is dissociated from target detection in human posterior parietal cortex. Nat Neurosci 3:292-297.

Corbetta M, Tansy AP, Stanley CM, Astafiev SV, Snyder AZ, Shulman GL (2005) A functional MRI study of preparatory signals for spatial location and objects. Neuropsychologia 43:2041-2056.

Desimone R, Duncan J (1995) Neural mechanisms of selective visual attention. Annu Rev Neurosci 18:193-222.

Duncan J, Humphreys G, Ward R (1997) Competitive brain activity in visual attention. Curr Opin Neurobiol 7:255-261.

Ellison A, Lane AR, Schenk T (2007) The interaction of brain regions during visual search processing as revealed by transcranial magnetic stimulation. Cereb Cortex 17:2579-2584.

Giesbrecht B, Woldorff MG, Song AW, Mangun GR (2003) Neural mechanisms of top-down control during spatial and feature attention. Neuroimage 19:496-512.

Giesbrecht B, Weissman DH, Woldorff MG, Mangun GR (2006) Pre-target activity in visual cortex predicts behavioral performance on spatial and feature attention tasks. Brain Res 1080:63-72.

Grefkes C, Fink GR (2005) The functional organization of the intraparietal sulcus in humans and monkeys. J Anat 207:3-17.

Heinze HJ, Mangun GR, Burchert W, Hinrichs H, Scholz M, Münte TF, Gös A, Scherg M, Johannes S, Hundeshagen H (1994) Combined spatial and temporal imaging of brain activity during visual selective attention in humans. Nature 372:543-546.

Hopfinger JB, Buonocore MH, Mangun GR (2000) The neural mechanisms of top-down attentional control. Nat Neurosci 3:284-291.

Kastner S, Ungerleider LG (2000) Mechanisms of visual attention in the human cortex. Annu Rev Neurosci 23:315-341.

Livingstone MS, Hubel DH (1987) Psychophysical evidence for separate channels for the perception of form, color, movement, and depth. J Neurosci 7:3416-3468.

Luks TL, Simpson GV (2004) Preparatory deployment of attention to mo- 
tion activates higher-order motion-processing brain regions. Neuroimage 22:1515-1522.

Macaluso E, Eimer M, Frith CD, Driver J (2003) Preparatory states in crossmodal spatial attention: spatial specificity and possible control mechanisms. Exp Brain Res 149:62-74.

Maunsell JH, Treue S (2006) Feature-based attention in visual cortex. Trends Neurosci 29:317-322.

Morris AP, Chambers CD, Mattingley JB (2007) Parietal stimulation destabilizes spatial updating across saccadic eye movements. Proc Natl Acad Sci U S A 104:9069-9074.

Orban GA, Claeys K, Nelissen K, Smans R, Sunaert S, Todd JT, Wardak C, Durand JB, Vanduffel W (2006) Mapping the parietal cortex of human and non-human primates. Neuropsychologia 44:2647-2667.

Pascual-Leone A, Walsh V (2001) Fast backprojections from the motion to the primary visual area necessary for visual awareness. Science 292:510-512.

Perry RJ, Zeki S (2000) The neurology of saccades and covert shifts in spatial attention: an event-related fMRI study. Brain 123:2273-2288.

Posner MI, Snyder CR, Davidson BJ (1980) Attention and the detection of signals. J Exp Psychol 109:160-174.

Rorden C, Brett M (2000) Stereotaxic display of brain lesions. Behav Neurol 12:191-200.

Ruff CC, Blankenburg F, Bjoertomt O, Bestmann S, Freeman E, Haynes JD, Rees G, Josephs O, Deichmann R, Driver J (2006) Concurrent TMSfMRI and psychophysics reveal frontal influences on human retinotopic visual cortex. Curr Biol 16:1479-1488.

Ruff CC, Bestmann S, Blankenburg F, Bjoertomt O, Josephs O, Weiskopf N, Deichmann R, Driver J (2008) Distinct causal influences of parietal versus frontal areas on human visual cortex: evidence from concurrent TMS fMRI. Cereb Cortex 18:817-827.

Rushworth MF, Taylor PC (2006) TMS in the parietal cortex: updating representations for attention and action. Neuropsychologia 44:2700-2716.

Saalmann YB, Pigarev IN, Vidyasagar TR (2007) Neural mechanisms of visual attention: how top-down feedback highlights relevant locations. Science 316:1612-1615.

Sack AT, Kohler A, Bestmann S, Linden DE, Dechent P, Goebel R, Baudewig J (2007) Imaging the brain activity changes underlying impaired visuospatial judgements: simultaneous fMRI, TMS, and behavioral studies. Cereb Cortex 17:2841-2852.

Saenz M, Buracas GT, Boynton GM (2002) Global effects of feature-based attention in human visual cortex. Nat Neurosci 5:631-632.
Schoenfeld MA, Hopf JM, Martinez A, Mai HM, Sattler C, Gasde A, Heinze HJ, Hillyard SA (2007) Spatio-temporal analysis of feature-based attention. Cereb Cortex 17:2468-2477.

Serences JT, Boynton GM (2007) Feature-based attentional modulations in the absence of direct visual stimulation. Neuron 55:301-312.

Serences JT, Yantis S (2007) Spatially selective representations of voluntary and stimulus-driven attentional priority in human occipital, parietal, and frontal cortex. Cereb Cortex 17:284-293.

Shulman GL, Ollinger JM, Akbudak E, Conturo TE, Snyder AZ, Petersen SE, Corbetta M (1999) Areas involved in encoding and applying directional expectations to moving objects. J Neurosci 19:9480-9496.

Slagter HA, Kok A, Mol N, Kenemans JL (2005a) Spatio-temporal dynamics of top-down control: directing attention to location and/or color as revealed by ERPs and source modeling. Brain Res Cogn Brain Res 22:333-348.

Slagter HA, Kok A, Mol N, Talsma D, Kenemans JL (2005b) Generating spatial and nonspatial attentional control: an ERP study. Psychophysiology 42:428-439.

Slagter HA, Giesbrecht B, Kok A, Weissman DH, Kenemans JL, Woldorff MG, Mangun GR (2007) fMRI evidence for both generalized and specialized components of attentional control. Brain Res 1177:90-102.

Treisman AM, Gelade G (1980) A feature-integration theory of attention. Cognit Psychol 12:97-136.

Treue S, Martinez-Trujillo JC (2007) Attending to features inside and outside the spotlight of attention. Neuron 55:174-176.

Vallar G, Perani D (1986) The anatomy of unilateral neglect after righthemisphere stroke lesions. A clinical/CT-scan correlation study in man. Neuropsychologia 24:609-622.

Vandenberghe R, Gitelman DR, Parrish TB, Mesulam MM (2001) Location- or feature-based targeting of peripheral attention. Neuroimage 14:37-47.

Woldorff MG, Hazlett CJ, Fichtenholtz HM, Weissman DH, Dale AM, Song AW (2004) Functional parcellation of attentional control regions of the brain. J Cogn Neurosci 16:149-165.

Womelsdorf T, Anton-Erxleben K, Pieper F, Treue S (2006) Dynamic shifts of visual receptive fields in cortical area MT by spatial attention. Nat Neurosci 9:1156-1160.

Yantis S, Serences JT (2003) Cortical mechanisms of space-based and object-based attentional control. Curr Opin Neurobiol 13:187-193. 